\title{
Research on Reconstruction Method for Illumination Field based on CMAC Neural Network ${ }^{*}$
}

\author{
Qian-sheng Fang, Jun Huang, Zhen-ya Zhang \\ Anhui provincial Key Laboratory of Intelligent Building, Anhui Jianzhu University, Hefei 230022, China \\ E-mail: zzychm@ustc.edu.cn or yangzhuhj@sina.com
}

\begin{abstract}
In intelligent building applications, the awareness of built environment illumination filed representatively applies in intelligent lighting systems for providing decision and support. In fact, the dynamic analysis of the built environment can assist in the illumination field of security systems at the same time can also work as a new means of achieving safety precautions. In this paper, a simple interest in the built environment based on discrete data at the point of illumination perception of the built environment illumination field reconstruction method is proposed based on the fast reconstruction method CMAC neural network architecture space illumination field. Experimental results show that with the use of a typical area of interpolation, polynomial interpolation, inverse distance weighting interpolation method reconstructed building space illumination field compared to the use of constructed building space reconstruction method based on CMAC illumination field of architectural space illumination field high precision.
\end{abstract}

Keywords-illumination; illumination field; CMAC neural network; reconstruction

\section{INTRODUCTION}

Illumination is the built environment, one of the built environment parameters especially close attention to the construction of intelligent environments researchers[1]. Illumination is used to indicate the extent of the built environment as well as the strength of the surface area of illumination of the object to be illuminated for relating applications in intelligent buildings. Typically, it can realize the building lighting system of intelligent controlled by detecting the changes of building environmental illumination, by the favor of the energy while improving human settlements convenience and comfort of the environment[2 4]. At the same time, the security video monitoring system, through the environmental illumination detection in the building changes can be timely to fill the light to monitor the area[5,6], which is conducive to the normal operation of security video surveillance system.

Taking into account the use of illumination is the space objects represented by quantized illumination level, if we can describe carefully the degree of space lighted level by a certain time in the architectural space illumination field which the building space and illumination in the relevant events and situations judged by the changes in the building illumination field. The judge of the building space's events and situations relevant illumination based on event identification can provide new data for related application of the intelligent building and intelligent home[7 11].

Wireless sensor network can facilitate effective deployment location acquisition illumination sensors data by deploying the right amount of space in the building illumination sensor[12 15].According to discrete illumination values at the point of interest for reconstructing the illumination field, which is a prerequisite to determine the changes in the building illumination field of building space for the relevant events and situations. Area interpolation, polynomial, inverse distance weighted interpolation methods are used to reconstruct illumination field by the discrete points of illumination value[16]. In order to reconstruct the illumination field quickly, this paper designs the space illumination field reconstruction using CMAC neural network method. Experimental results show that the method can reconstruct quickly and accurately the illumination field based on the discrete points of illumination value.

\section{ILLUMINATION RECONSTRUCTED BASED ON CMAC NEURAL NETWORK}

\section{A. The topology structure of CMAC neural network}

CMAC network is a kind of simulating cerebellar function and neural network associative function (Cerebellar Model Articulation Controller, abbreviated CMAC ) within strong generalization ability; The output of the network only need to participate in the calculation amount of neurons and concept mapping layer, the actual mapping layer. The CMAC neural network use $\delta$ training algorithm which has fast convergence speed and is no local minimum point problem.

In intelligent building applications, we need to take into account the negative impact of the deployment of the sensor function of the building as well as minimizing the convenience sensor deployment that all illumination sensors may be deployed in the same plane. At this point, we can use projective geometry method to make an analysis the planar illumination field for achieving events and situation related to the building's illumination. The topological structure of the CMAC neural network reconstruction plane illumination field is showed in Fig.1. 


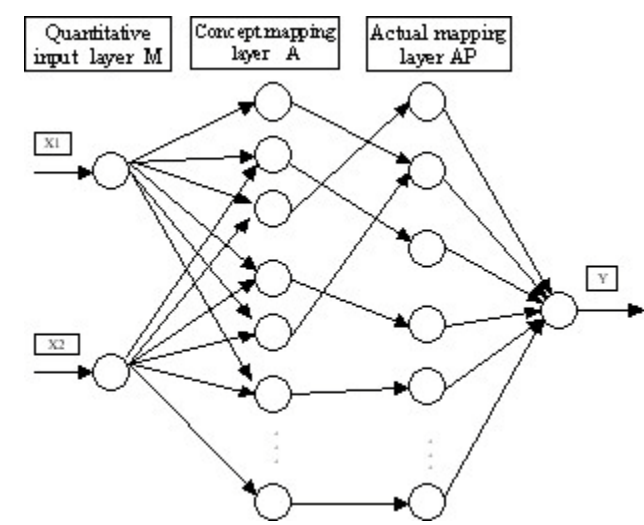

Fig. 1. Topology of CMAC neural network for illumination field reconstruction

Fig.1 shows the CMAC neural network within one input and two output which is the composition of three layer structure with quantitative input layer $\mathrm{M}$, concept mapping A and actual mapping layer AP and the value of generalization parameter is six and the value of quantitative precision is ten. The input of $\mathrm{x}=(\mathrm{x} 1, \mathrm{x} 2)$ by quantitative $\mathrm{M}$ is quantified the value of the input layer and transports each layer of quantify the value to the concept mapping memory cell address; AP layer uses Hash technology to address mapping from $\mathrm{A}$ to take random small address space; the output neurons are activated neurons in layer AP of the weighted cumulative output as the network output of Y.

\section{B. Realize illumination field reconstruction method based on CMAC neural network}

The CMAC neural network is used to reconstruct the plane illumination field is divided into the training process of CMAC neural network and the use of the trained CMAC neural network calculation of plane anywhere in the illumination of the two sub processes. Algorithm 1 and Algorithm 2 give the form description of the two sub processes.

\section{Algorithm 1 :for the illumination field reconstruction CMAC neural network training}

Input: the observation plane of illumination sequence in the $\mathrm{S}=\left\{\left(\mathrm{x}_{\mathrm{i}}, \mathrm{y}_{\mathrm{i}}, \mathrm{l}_{\mathrm{i}}\right) \mid \mathrm{i}=1,2 \ldots \mathrm{N}\right\} ; \mathrm{D}=[\mathrm{a} \mathrm{b}] \mathrm{x}[\mathrm{c} \mathrm{d}]$ region, the generalization of $\mathrm{C}$, quantization precision constant learning rate of delta, Q, maxN and iteration error e ;

Output: CMAC neural network trained by net

1) input normalized structure of $S\left(S^{\prime}=\left\{x_{i}^{\prime}, y_{i}^{\prime}, l_{i}^{\prime}\right) \mid i=1,2 \ldots N\right\}$;

2) quantify horizontal and vertical coordinates of the of the input, get each quantized value activity quantization storage unit, use floor () function to encode mapped address; get the weight storage unit;

3) use generalization of constant $\mathrm{C}$, quantization $\mathrm{Q}$, learning rate of delta to construct the 2 input and 1 output CMAC neural network net;
4) $\mathrm{k}=0$ error $=2 * \mathrm{e}$;

5) while $\mathrm{k}<=\operatorname{maxN}$ and error $<\mathrm{e}$;

6) output in the quantized $\mathrm{S}^{\prime}$ as input to calculate net;

7) input error calculation of error network;

8) use the $\delta$ algorithm of the network weights adjustment;

9) $\mathrm{k}=\mathrm{k}+1$;

10) CMAC neural network trained by net output.

\section{Algorithm 2: illumination computing method based on CMAC neural network}

Input: CMAC neural network net; $\mathrm{D}=[\mathrm{a} \mathrm{b}] \mathrm{x}[\mathrm{c} \mathrm{d}], \mathrm{C}, \mathrm{Q}$, the generalization of constant quantization precision; $(\mathrm{x}, \mathrm{y})$ and $\mathrm{D}$;

Output: the illumination value of point $(\mathrm{x}, \mathrm{y})$

1) $(x, y)$ will be normalized $\left(x^{\prime}, y^{\prime}\right)$;

2) $(x, y)$ will be quantified for $\left(x^{\prime}, y^{\prime}\right)$, calculating the value of the storage unit;

3) use the net to calculated the illumination value 1 of $\left(x^{\prime}, y^{\prime}\right)$;

4) return 1 as the illumination value of $(x, y)$.

Step7 of algorithm 1 needs to calculate value of CMAC neural network output. We can use the absolute error of TAE (Total Absolute Error) and root mean square error of RMSE (Root Mean Square Error). We can set up the output sequence as $\left\{\mathrm{z}_{1}, \mathrm{Z}_{2}, \ldots, \mathrm{Z}_{\mathrm{n}}\right\}$ and the actual output sequence as $\left\{z_{1}, z_{2}, \ldots, z_{n}\right\}$, which use the type (1) and type(2) to calculate the value of TAE and RMSE. Taking into account the rapid demand computing, computing root mean square error of the RMSE can be used for type (3) the provisions of MSE instead of the mean square error.

$$
\begin{array}{r}
\text { TAE }=\sum_{i=1}^{n}\left|z_{i}-z_{i}^{\prime}\right| \\
R M S E=\sqrt{\frac{1}{n} \sum_{i=1}^{n}\left(z_{i}-z_{i}^{\prime}\right)^{2}} \\
M S E=\frac{1}{n} \sum_{i=1}^{n}\left(z_{i}-z_{i}^{\prime}\right)^{2}
\end{array}
$$

\section{EXPERIMENTAL RESULTS AND ANALYSIS}

In order to test the validity of algorithm 1,2 reconstruction of illumination in building space, experiments use glass to build a simple model of architectural space model within 1 meters long, 0.5 meters wide, 0.4 meters high, the bottom for the board and the possible moving around glass door; It gives the part of the glass full of shading treatment by using black foam sheet as the shading material. In the experiment, the illumination sensors complete 1 times per second acquisition interest point illumination tasks by arranging on nine points of 
interest model floor. To test the validity of reconstruct the illumination field of the model's bottom surface, experiments take illumination data acquisition at 5 points of interest as the sample data to train the CMAC neural network, the other 4 interest points illumination data as test data.

Firstly, the experiment uses the illumination sensors to collect the all interest points of 50 groups of illumination data and respectively uses polynomial interpolation, inverse distance weighted interpolation method, area interpolation of three kinds interpolation methods as well as algorithm 1 and algorithm 2 of the plane illumination field reconstruction method based on the bottom surface of the illumination field calculation model. Each illumination field reconstruction method of TAE and RMSE were calculated using the test data. Fig.2 gives four kinds of methods of 50 times reconstruction model bottom illumination field absolute error of TAE distribution histogram and TABLE I gives the relevant descriptions. Obviously, whether it considers the minimum value, maximum value and mean of TAE or considers the variance of TAE, the illumination field reconstruction method has better performance based on CMAC neural network.

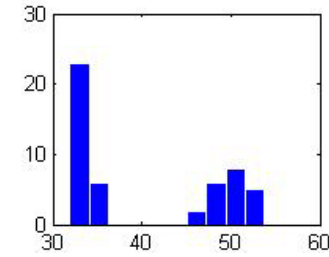

(a)polynomial interpolation

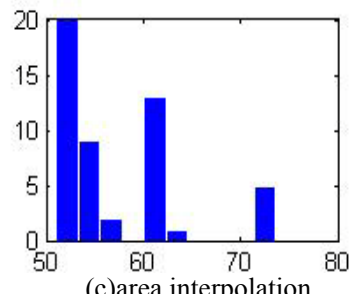

(c)area interpolation

Fig. 2. TAE histogram of illumination field reconstruction

TABLE I.

TAE STATISTICAL OF ILLUMINATION FIELD RECONSTRUCTION

\begin{tabular}{|c|c|c|c|c|}
\hline & $\begin{array}{c}\text { polynomial } \\
\text { interpolation }\end{array}$ & $\begin{array}{c}\text { inverse } \\
\text { distance } \\
\text { weighted }\end{array}$ & $\begin{array}{c}\text { area } \\
\text { interpolation }\end{array}$ & $\begin{array}{c}\text { CMAC } \\
\text { neural } \\
\text { network }\end{array}$ \\
\hline maximum & 53.8035 & 56.9341 & 73.6625 & 32.5682 \\
\hline minimum & 31.9060 & 29.6425 & 51.0150 & 17.4524 \\
\hline mean & 40.4264 & 39.1373 & 57.2767 & 23.2626 \\
\hline variance & 8.4577 & 9.3469 & 6.5760 & 5.4450 \\
\hline
\end{tabular}

Similarly, Fig. 3 gives four kinds of methods of 50 times reconstruction model bottom illumination field root mean square error of RMSE distribution histogram and TABLE II gives the relevant descriptions. Obviously, whether it considers the minimum value, maximum value and mean of RMSE or considers the variance of RMSE, the illumination field reconstruction method has better performance based on CMAC neural network.

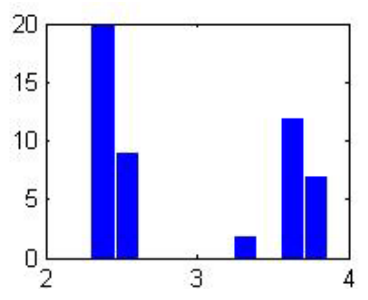

(a)polynomial interpolation

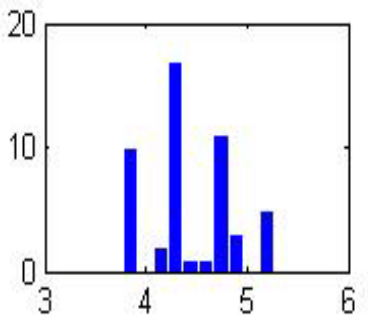

(c)area interpolation

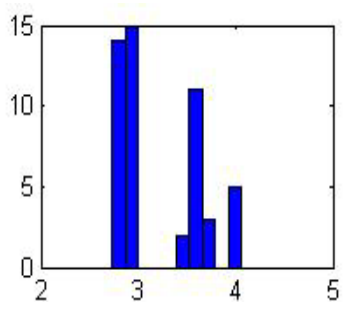

(b)inverse distance weighted
Fig. 3. RMSE histogram of illumination field reconstruction

TABLE II. RMSE OF ILLUMINATION FIELD RECONSTRUCTION

\begin{tabular}{|c|c|c|c|c|}
\hline & $\begin{array}{c}\text { polynomial } \\
\text { interpolation }\end{array}$ & $\begin{array}{c}\text { inverse } \\
\text { distance } \\
\text { weighted }\end{array}$ & $\begin{array}{c}\text { area } \\
\text { interpolation }\end{array}$ & $\begin{array}{c}\text { CMAC } \\
\text { neural } \\
\text { network }\end{array}$ \\
\hline maximum & 3.8675 & 4.0564 & 5.2639 & 2.6416 \\
\hline minimum & 2.2991 & 2.7317 & 3.7709 & 1.6345 \\
\hline mean & 2.9384 & 3.2225 & 4.4335 & 2.2506 \\
\hline variance & 0.6176 & 0.4251 & 0.4193 & 0.3276 \\
\hline
\end{tabular}

\section{CONCLUSION AND OUTLOOK}

Illumination is one of the most important environment parameters in buildings. On the one hand, it can provide decision support for intelligent illumination system by perceiving the single or multiple discrete positions' illumination data in environment of building; on the other hand, it can be used as a kind of the new security means. This paper studies the method of reconstruction of building environmental illumination field based on discrete points of interest the perception of simple construction environment and puts forward a fast reconstruction method of architectural space illumination field based on CMAC neural network. Experiments show the architectural space illumination field reconstruction method has a high precision based on CMAC neural network.

The reconstruction method of CMAC neural network architecture space illumination field based on CMAC neural network is trained by the $\delta$ algorithm. In order to promote 
the use of building space illumination field reconstruction method of architectural space illumination precision based on CMAC neural network which the learning method need further study in future. At the same time, we need to pay attention to design the security system based on illumination field's monitoring and analysis which combine with the security specific needs.

\section{ACKNOWLEDGMENT}

This work is partially funded by National Key Technology Research and Development Program of the Ministry of Science and Technology of China (2012BAJ08B01), the National Natural Science Foundation of China (11471304, 61340030, 61300060), Science and technology project of Ministry Housing and Urban-Rural Development Department(2014-K8-061), the Special Foundation for Young Scientists of Anhui Province (2013SQRL043ZD) and the Natural Science Foundation of Anhui Province (1508085QF131).

\section{REFERENCE}

[1] Yuwei Zhang. Wireless monitoring network system for indoor light environment[D].Zhejiang University,2012.

[2] Niels van de Meugheuvel, Ashish Pandharipande, David Caicedo. Distributed lighting control with daylight and occupancy adaptation.Energy and Buildings, Volume 75, June 2014, Pages 321329.

[3] Siriwarin Petcharat, Supachart Chungpaibulpatana, Pattana Rakkwamsuk. Assessment of potential energy saving using cluster analysis: A case study of lighting systems in buildings. Energy and Buildings, Volume 52, September 2012, Pages 145-152.

[4] Mohammad Asif ul Haq, Mohammad Yusri Hassan, Hayati Abdullah. A review on lighting control technologies in commercial buildings, their performance and affecting factors .Renewable and Sustainable Energy Reviews, Volume 33, May 2014, Pages 268-279.

[5] Wenfeng Zhang. Application of LED lights in the video monitoring system[J]. Chinese new communication,2012,16:53-54

[6] Wei Ye, Tianrong You, Shaohua Shen. Application of high-definition video surveillance light technology [J],Chinese security,2014,13:75-78.

[7] Ping Jia, Ning Xu, Ye Zhang. Automatic target recognition based on local feature extraction[J]. Optics and Precision Engineering,2013,07:1898-1905.

[8] Xiaoxin Ren, Da Yan.A study of lighting energy consumption model for office buildings based on occupant behavior [J].Building Science,2014,06:1-9+18.

[9] Sara Rodríguez, Juan F. De Paz, Gabriel Villarrubia. Multi-Agent Information Fusion System to manage data from a WSN in a residential home.Information Fusion, Volume 23, May 2015, Pages 43-57.

[10] Haichao Ran, Lihua Sun, Xiaozhi Gao. Influences of intelligent evacuation guidance system on crowd evacuation in building fire. Automation in Construction, Volume 41, May 2014, Pages 78-82.

[11] Yang, Hanhua. A reliable wireless sensor network multi path routing scheme for environmental monitoring system. Information Technology Applications in Industry,Applied Mechanics and Materials, v 263-266, n PART 1, p 959-962, 2013.

[12] E.S. Nadimi, R.N. Jorgensen, V. Blanes -Vidal. Monitoring and classifying animal behavior using ZigBee-based mobile ad hoc wireless sensor networks and artificial neural networks. Computers and Electronics in Agriculture, Volume 82, March 2012, Pages 44-54.

[13] Charalambos Sergiou, Vasos Vassiliou. Congestion control in Wireless Sensor Networks through dynamic alternative path selection. Computer Networks, Volume 75, Part A, 24 December 2014, Pages 226-238.
[14] Guojian Wang, Junmin Bao, Sang Li ,et al. Research and realization of distributed monitorinf system environment illumination $[\mathrm{J}]$. Automation Instrumentation,2013,09:34-36.

[15] JinMin Choi, Hyung Jin Chang, Yung Jun Yoo. Robust moving object detection against fast illumination change. Computer Vision and Image Understanding, Volume 116, Issue 2, February 2012, Pages 179-193.

[16] Minggang Jing, Jitao Wu. Fast image interpolation using directional inverse distance weighting for real-time applications. Computers \& Geosciences, Volume 34, Issue 9, September 2008, Pages 1044-1055. 